\title{
The role of information in complex systems \\ Self-organisation in stem cells and glass formers
}

\author{
Karoline Wiesner \\ School of Mathematics \\ University of Bristol
}

4th International Electronic Conference on Entropy and Its Applications November 2017 


\section{One property of complex systems: structure on the macroscopic level which does not exist on the microscopic level $\left(^{*}\right)$}

$\left(^{*}\right)$ for more on features of complex systems, see J Layman and K Wiesner, What is a complex system?, book to be published in 2018 


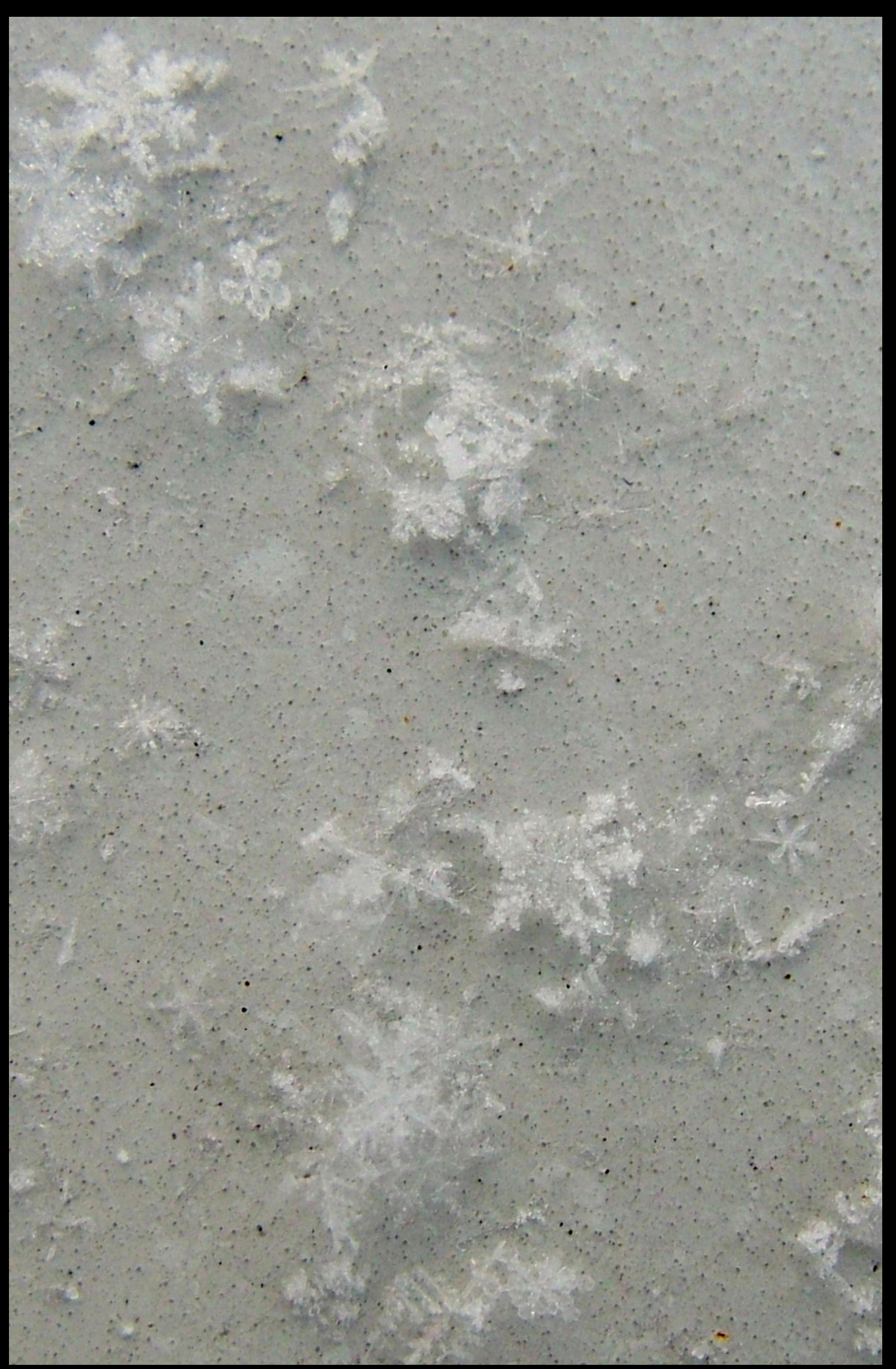

"A snow crystal at -110 C" by By En-cas-de-soleil is licensed under CC BY-SA 4.0

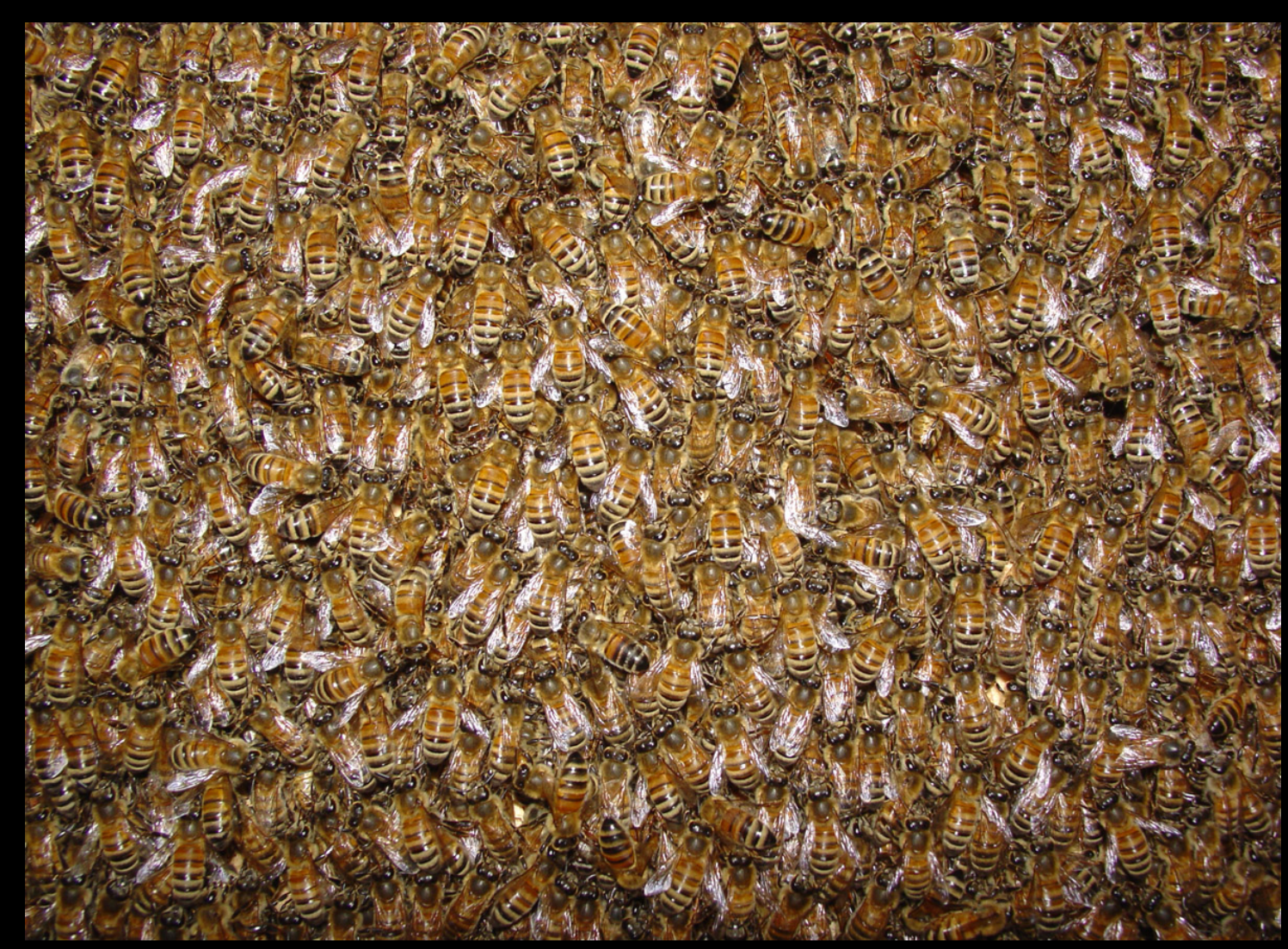

"bees" by alan taylor is licensed under CC BY 2.0

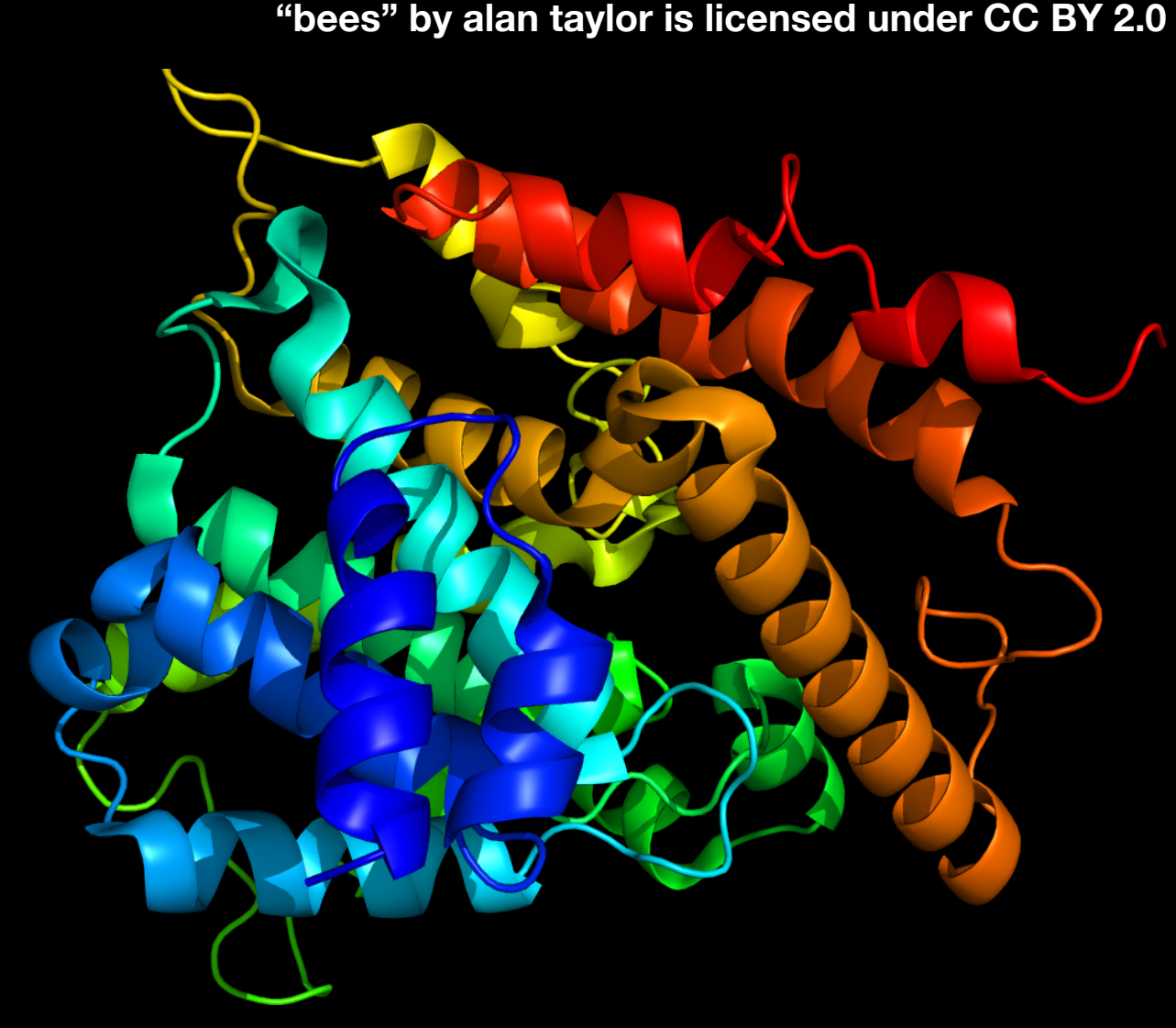

"Protein PDE8A PDB 1LHQ" by Pleiotrope is licensed under CC BY 2.0 


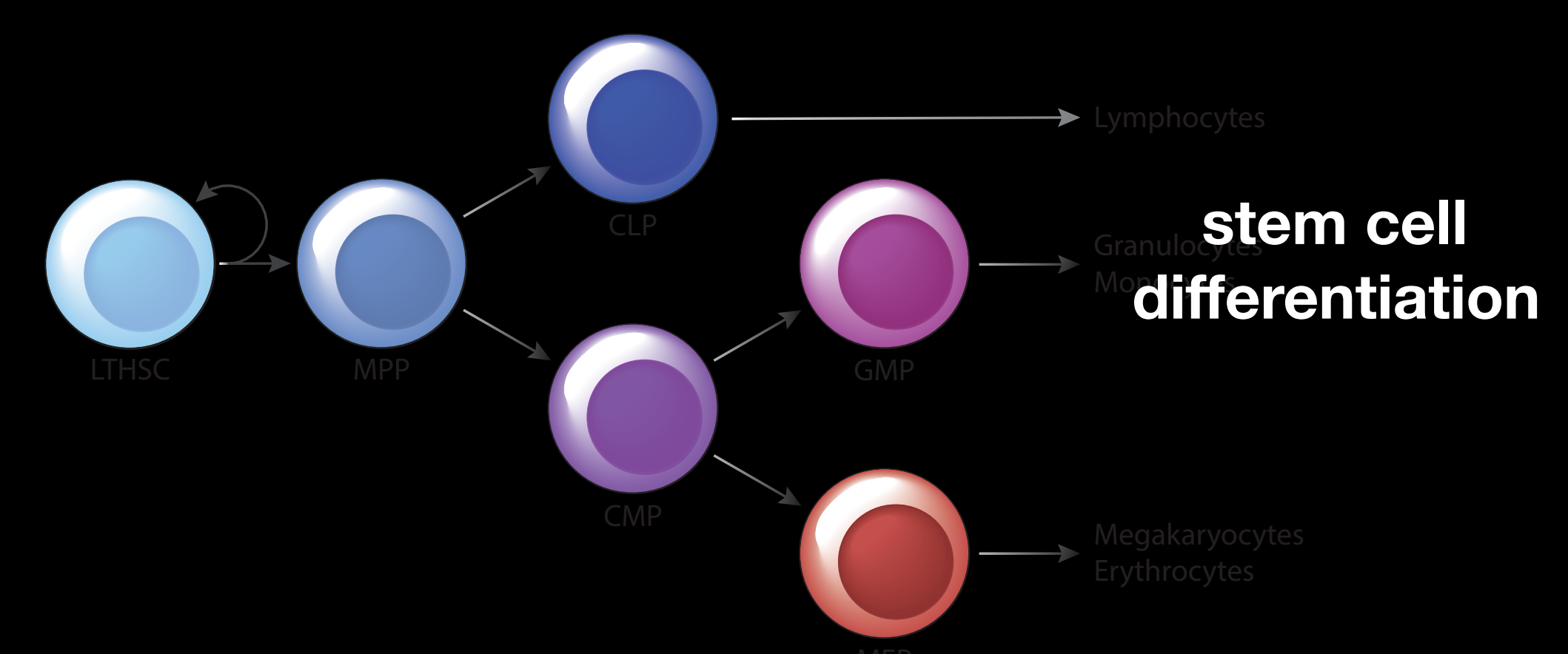

\section{Identifying moments and mechanisms of self-}

\section{organisation using entropy}

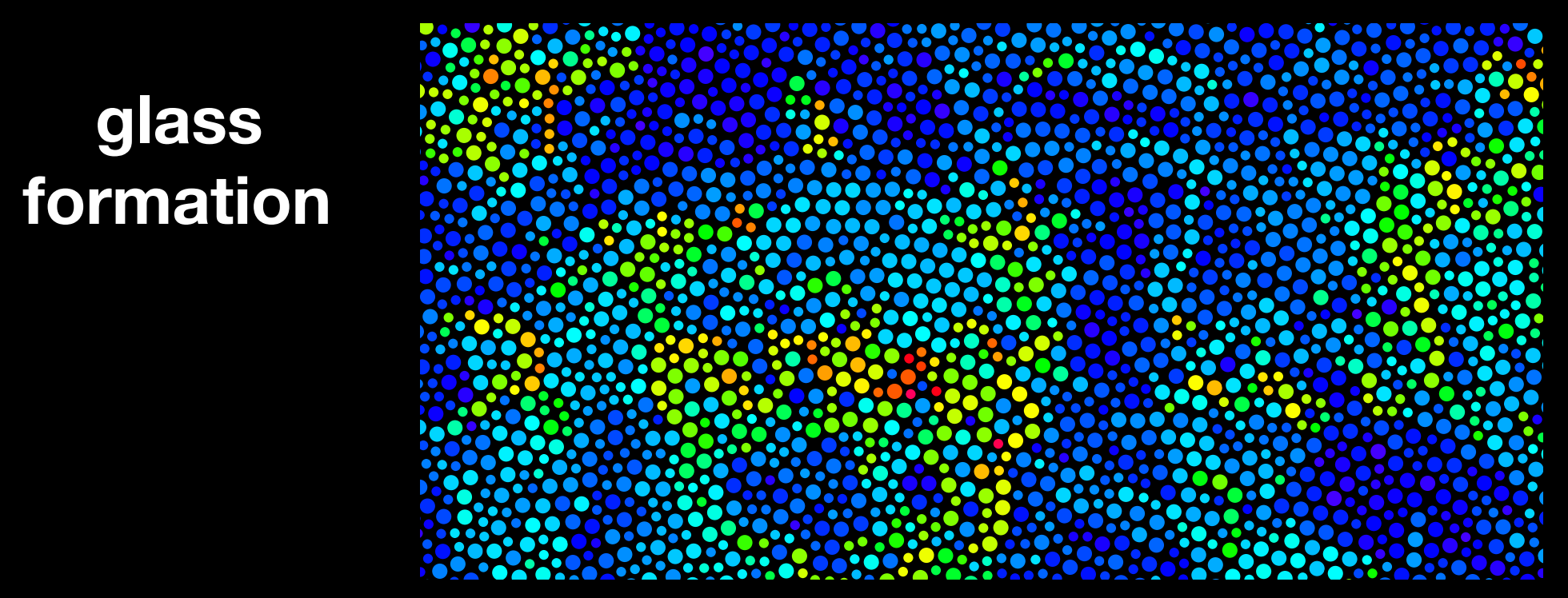




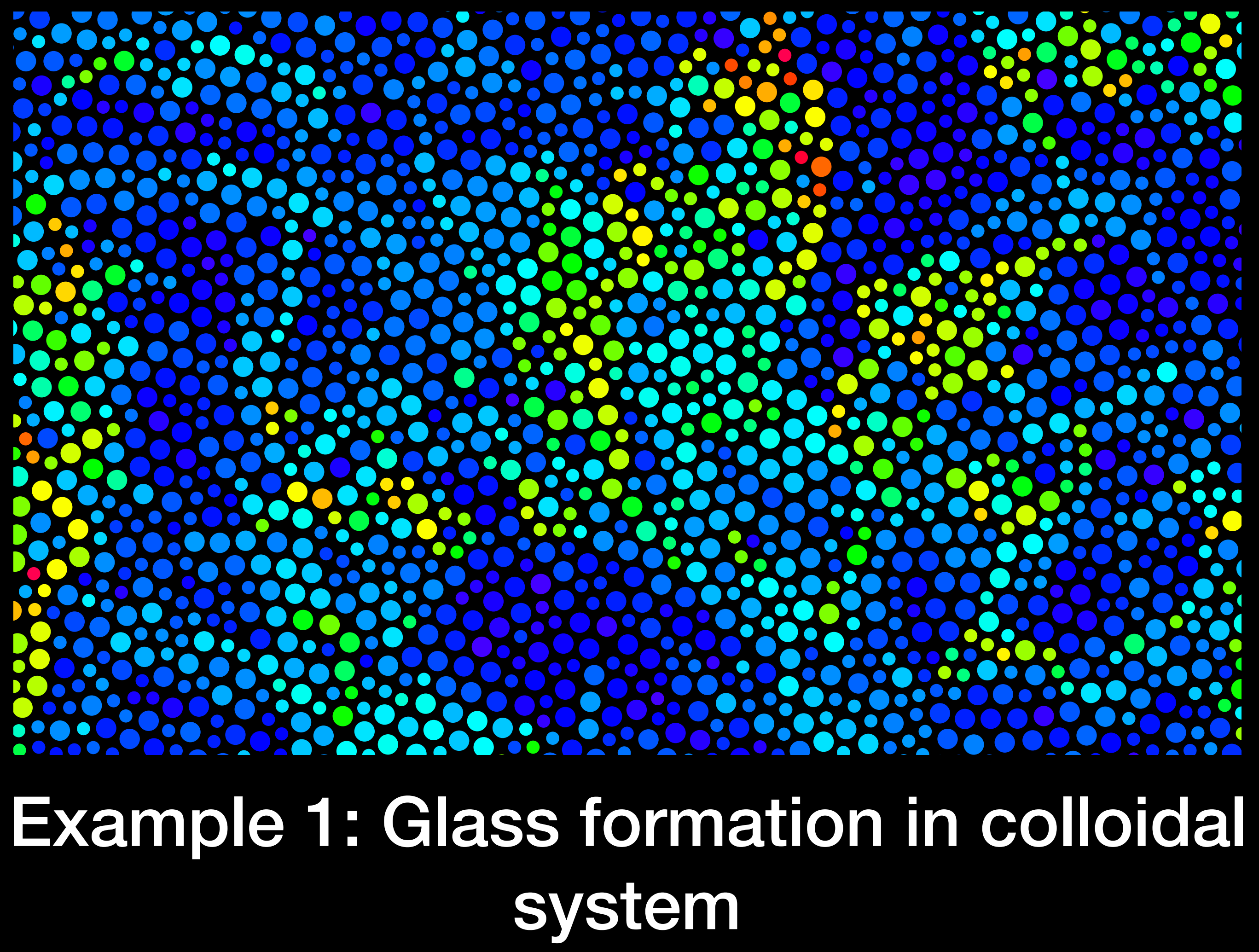




\section{Mutual Information Reveals Multiple Structural Relaxation Mechanisms in a Model Glass Former}

Dunleavy, Andrew J., Karoline Wiesner, Ryoichi Yamamoto, and C. Patrick Royall. Nature Communications 6 (January 2015). 


\section{Mutual information in a colloidal system}

number of correlated particles

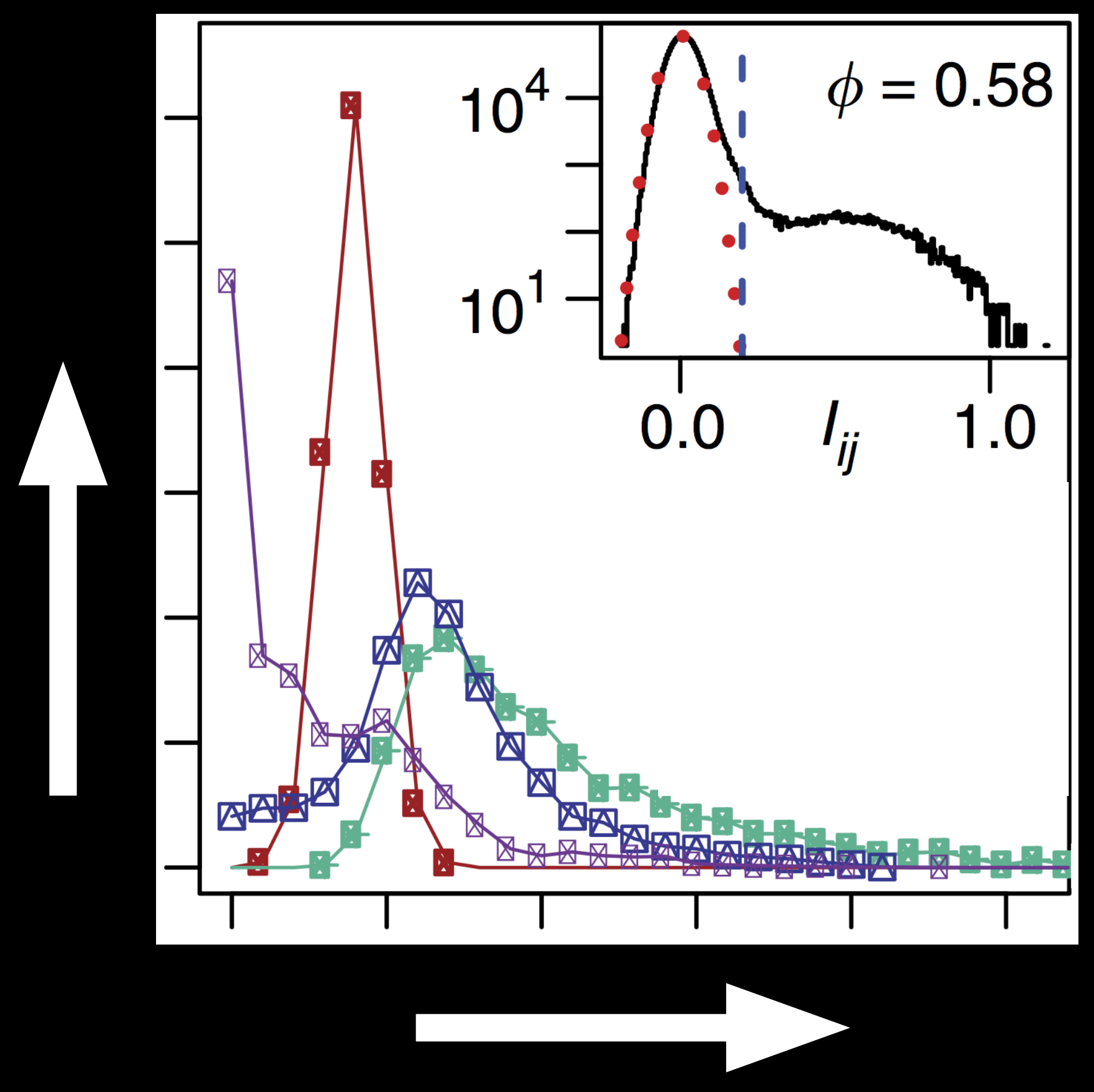

particles with high mutual information

time 


\section{Particles with significant mutual information}

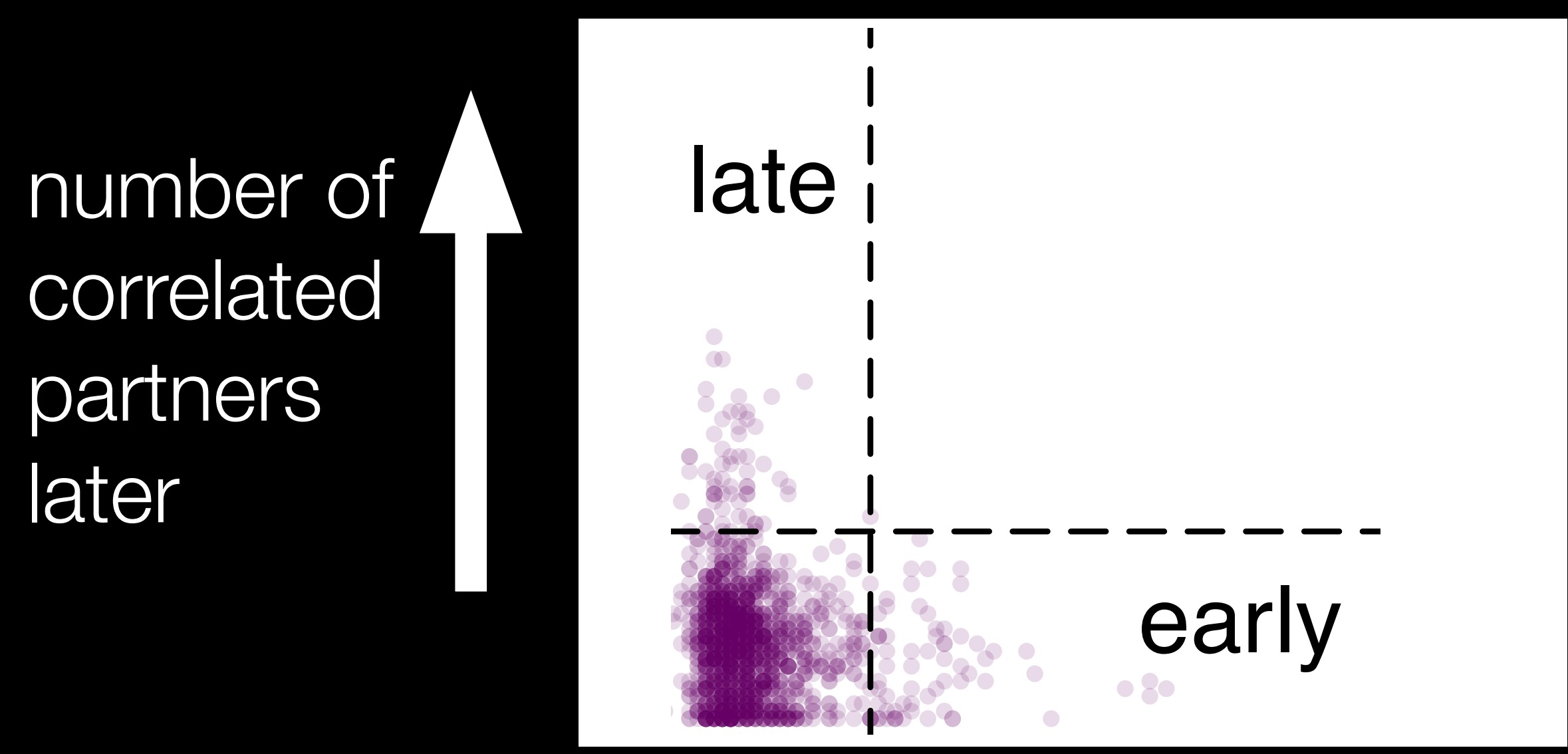

correlated partners later

number of correlated partners early on 


\section{Mutual information predicts major players in relaxation mechanism}

late movers

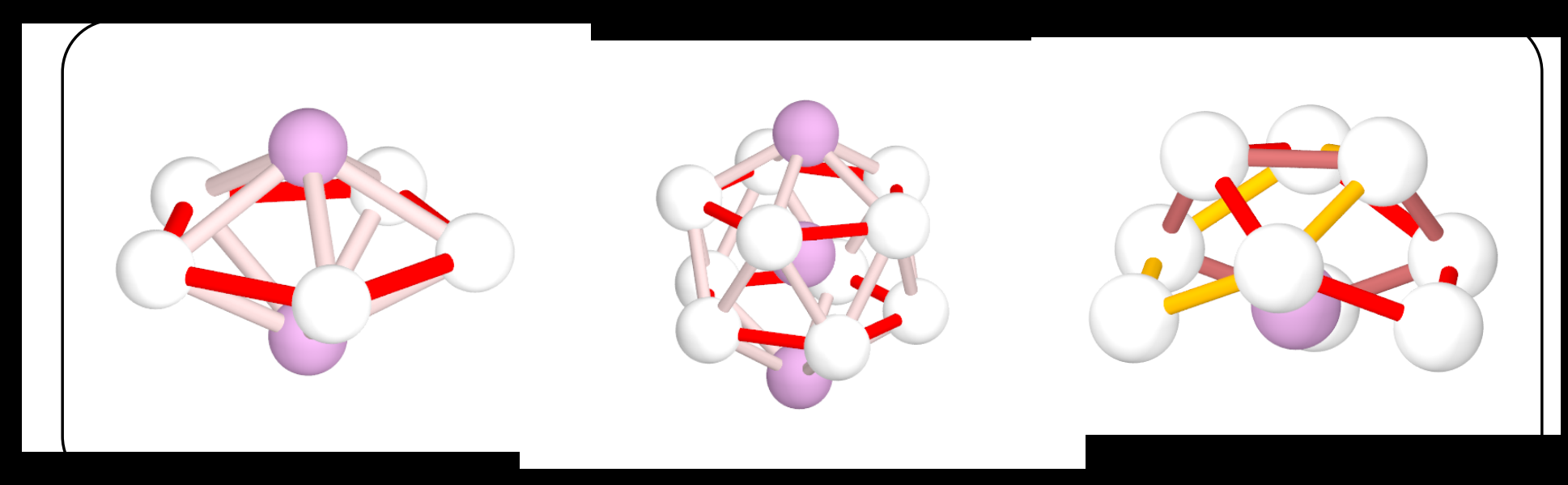

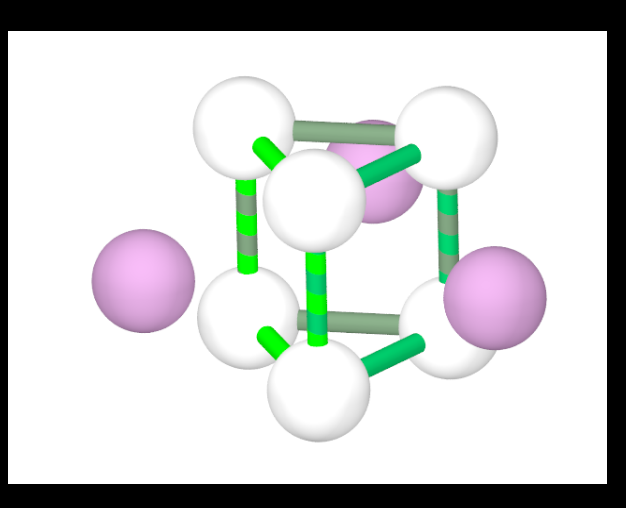

early

movers 


\section{New length scale, based on mutual information}

$$
I(\mathbf{r}, t)=\frac{\sum_{i j} I_{i j}(t) \delta\left(\mathbf{r}-\left|\mathbf{x}_{i}(0)-\mathbf{x}_{i}(0)\right|\right)}{\sum_{i j} \delta\left(\mathbf{r}-\left|\mathbf{x}_{i}(0)-\mathbf{x}_{i}(0)\right|\right)}
$$

Fit an exponential function to define the length scale $\xi_{\exp p}$ :

$$
I(\mathbf{r}, t) \propto e^{-\mathbf{r} / \xi_{\mathrm{e} x p}}
$$




\section{To summarise example 1}

- The mutual information between movements of particles did indicate where a structural transition was taking place.

- And it lead to a mechanistic explanation of the transition. 
Cellular potency

lineage

Example 1: Differentiating stem cell 


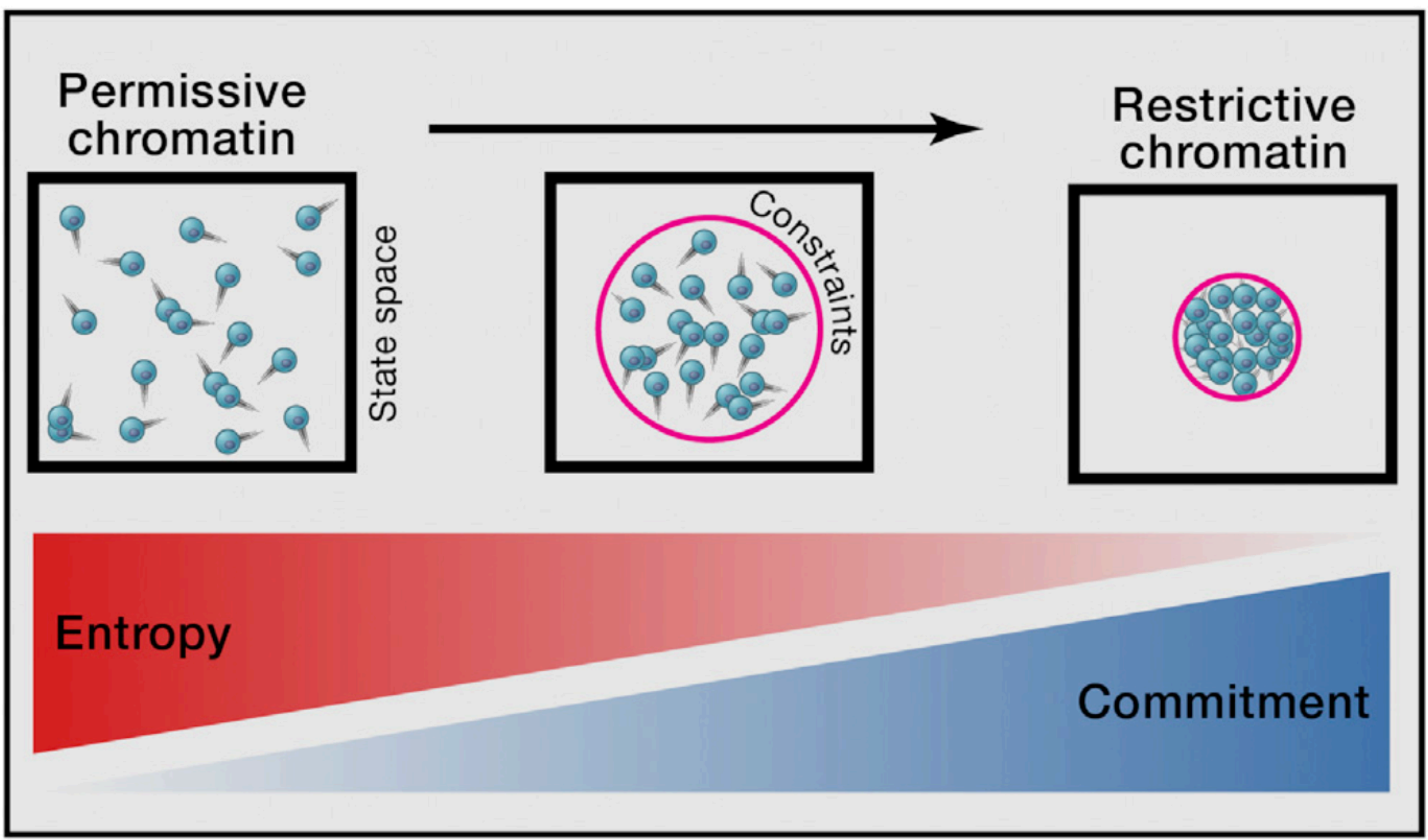

Figure 1. Entropy and Developmental Potency

Statistical mechanics analogy for stem cell development

from: MacArthur, B. D. \& Lemischka, I. R. Statistical Mechanics of Pluripotency. Cell 154, 484-489 (2013). 


\section{Hematopoietic stem cells - Entropic landscape of differentiation}

K. Wiesner, J. Teles, M. Hartnor, C. Petersen, arXiv.org, November 2017 


\section{Mapping Cellular Hierarchy} by Single-Cell Analysis of the Cell Surface Repertoire

Guoji Guo, ${ }^{1}$ Sidinh Luc, ${ }^{1}$ Eugenio Marco, ${ }^{3}$ Ta-Wei Lin, ${ }^{4}$ Cong Peng, ${ }^{1}$ Marc A. Kerenyi, ${ }^{1}$ Semir Beyaz, ${ }^{1}$ Woojin Kim, Jian Xu, ${ }^{1}$ Partha Pratim Das, ${ }^{1}$ Tobias Neff, ${ }^{5}$ Keyong Zou, ${ }^{6}$ Guo-Cheng Yuan, ${ }^{3}$ and Stuart H. Orkin ${ }^{1,2, *}$

${ }^{1}$ Division of Pediatric Hematology/Oncology, Boston Children's Hospital and Dana-Farber Cancer Institute, Harvard Stem Cell Institute, Harvard Medical School, Boston, MA 02115, USA

${ }^{2}$ Howard Hughes Medical Institute, Boston, MA 02115, USA

3Department of Biostatistics and Computational Biology, Dana-Farber Cancer Institute, Harvard School of Public Health, Boston,

MA 02115, USA

${ }^{4}$ Molecular Genetics Core Facility, Children's Hospital Boston, Boston, MA 02115, USA

${ }^{5}$ Pediatric Hematology/Oncology/BMT, University of Colorado, Aurora, CO 80045, USA

${ }^{6}$ Boston Open Labs, Cambridge, MA 02138, USA

${ }^{*}$ Correspondence: stuart_orkin@dfci.harvard.edu

http://dx.doi.org/10.1016/j.stem.2013.07.017

\section{Experimental data for entropy measurements}




\section{Entropy of gene expression}

- Binary random variable $\mathrm{X}$ :

$$
X=0 \text { if 'gene off', } X=1 \text { if 'gene on' }
$$

- Binary entropy:

$$
H(X)=-\sum_{x \in\{0,1\}} P(x) \log _{2} P(x)
$$




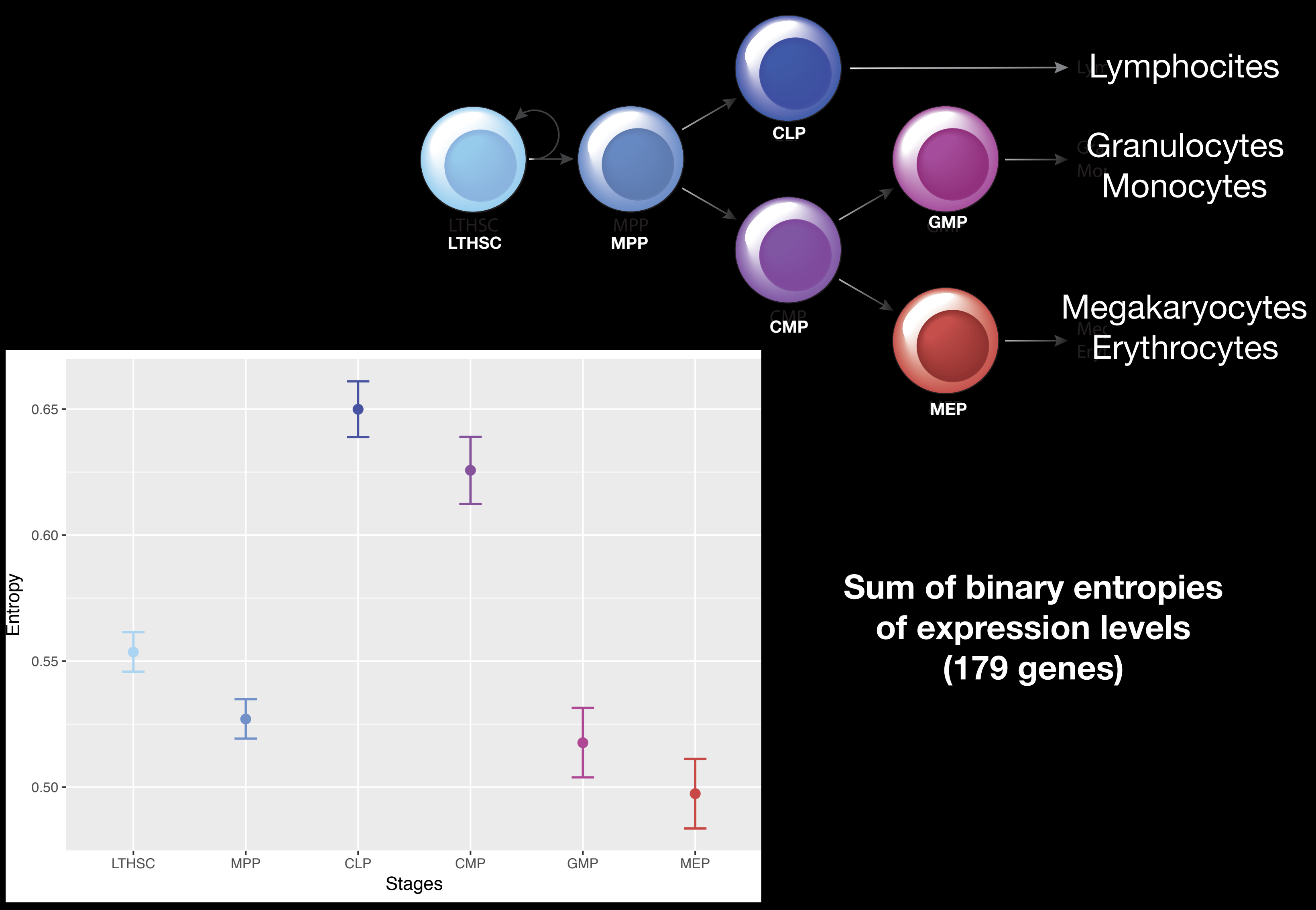




\section{To summarise example 2}

- Our observations are contrary to the expected continuous decrease of entropy. Instead we saw a significant increase in entropy during an intermediate stage before the entropy decreased, slightly below the initial value, in the last stages of the process as it was measured in these experiments. 


\section{Conclusions}

- The data of a liquid forming a glass were analysed using mutual information. We introduced a new length scale based on the mutual information. We were able to suggest a mechanism behind the glass forming process.

- The data of hematopietic stem cell differentiation was analysed using the Shannon entropy. Contrary to the general expectation, we found an increase in entropy toward the transition point before a decrease toward the final stages. 


\section{Acknowledgements}

- Andrew Dunleavy, Morgan Hartnor, James Ladyman, Carsten Petersen, Patrick Royall, Jose Teles, and Ryoichi Yamamoto

- EPSRC

- References:

Dunleavy, Andrew J., Karoline Wiesner, Ryoichi Yamamoto, and C. Patrick Royall. Nature Communications 6 (January 2015).

K. Wiesner, J. Teles, M. Hartnor, C. Petersen, arXiv.org, November 2017

J Ladyman, K. Wiesner (forthcoming) 